\title{
Case of focal cortical dysplasia in a tertiary rural hospital
}

\author{
Authors \\ Dr Hasnayan Sabir ${ }^{1}$, Dr Maria Tomy Prothasis ${ }^{2 *}$, Dr Aishwarya Ghule ${ }^{3}$, \\ Dr Sanyukta Hepat ${ }^{4}$, Dr Vidyashree Hulkoti ${ }^{5}$ \\ ${ }^{1}$ Assitant Professor, Dept. of Medicine, Jawaharlal Nehru Medical College, AVBR Hospital, DMIMS Univ., \\ Sawangi Meghe, Wardha \\ 2,3,4,5 Junior Residents, Dept. of Medicine, Jawaharlal Nehru Medical College, AVBR Hospital, DMIMS \\ Univ., Sawangi Meghe, Wardha,(M.S.) Pin : 442001 \\ Corresponding Author \\ Dr Maria Tomy Prothasis \\ Junior Resident, Dept. of Medicine, Jawaharlal Nehru Medical College, AVBR Hospital, \\ DMIMS Univ., Sawangi Meghe, Wardha (M.S.) Pin : 442001, India
}

\begin{abstract}
Focal cortical dysplasia (FCDs) which was considered to be a rare cause now it's being diagnosed easily due to better imaging facilities. It usually presents as focal seizures specially in pediatric population. In our case, 19-year-old girl presented with focal seizures since the age of five years. Electroencephalography (EEG) showed no significant abnormality. Magnetic resonance imaging (MRI) of Brain done revealed thickened cortex in right posterior parietal lobe, possibly focal cortical dysplasia with post ictal edema. Patient was then started on sodium valproate 500mg BD and dosage of carbamazepine was increased to 200mg BD after which her seizures were controlled after 2 days. In this case seizure was controlled by two anticonvulsants.
\end{abstract}

Keywords: Seizure, Focal cortical dysplasia, MRI, Anti convulsant.

\section{Introduction}

Focal cortical dysplasia (FCD) is a congenital abnormality of brain development in which intracranial neurons didn't migrate to proper formation in utero. This condition was originally described in 1971. Intractable seizures with mental retardation in later life are early symptoms and are associated with abnormal electroencephalographic (EEG) findings and MRI changes ${ }^{(1)}$. Cortical development malformations are a successive reason for symptomatic central seizures in children and adulthood. In particular, focal cortical dysplasia is progressively perceived in epilepsy patients inferable from improved MRI procedures ${ }^{(2)}$. Focal cortical dysplasia is the most widely recognized reason for therapeutically refractory seizures in the pediatric population. It is also the second or third most common cause of treatment resistant seizures in adults. It represents varied presentation of cortical malformations due to atypical patterns of brain development. In this, during normal advancement neurons neglect to spread outward to cerebral cortex and position effectively, so signals will misfire leading to seizure. It is highly correlated with childhood seizures syndrome as well as with 
cognitive disabilities and other neurological issues. Just couple of studies have so far concentrated their enthusiasm on clinical highlights in patients with FCD. There is just less information of the course of the illness. We report a case of focal intractable epilepsy with MRI suggestive of focal cortical dysplasia.

\section{Case Report}

A 19 year old girl born to a couple residing in village in district chandrapur presented to us for the first time with recurrent focal seizures since the age of five years. The antenatal and perinatal history was normal. The first seizure occurred at the age of five years of life when she is at home. That initially presented as focal seizures manifested with deviation of head to one side with uprolling of eye balls associated with tonic-clonic movements of right upper limb only. This was lasted for few seconds. She had been shifted to local hospital there and got investigated with CT scan and blood reports and treated with single anticonvulsant (carbamazepine $200 \mathrm{mg}$ OD). She was being diagnosed there as case of idiopathic epilepsy only as CT report was not that conclusive.EEG was done in May 2014 which was normal. Presently since the last eight days, focal seizure episodes in similar pattern as earlier and it has increased in frequency and were present in the day and night time. It has lasted for 1-2 minutes inspite of daily treatment. There was no h/o clenching of teeth, frothing from mouth, post ictal confusion, no bladder or faecal evacuation or tongue bite. There was no cutaneous or systematic abnormalities. General examination was within normal limits. Systemic examination including CNS examination was within normal limits. Patient admitted immediately and investigated. MRI brain done on May 2019(fig1\&2) showed thickened right posterior parietal lobe cortex with iso-intense signal onT1 and T2 weighed images and diffusion restriction and surrounding area of isointensity on DWI suggesting focal cortical dysplasia with post ictal edema. EEG done which was again normal. Patient was started with increased dosage of carbamazepine (200mg BD) initally dose increased to $200 \mathrm{mg}$ TDS but due to excessive giddiness dose has been reduced to 200 BD). Also another anticonvulsant sodium valproate $500 \mathrm{mg}$ BD has been added to terminate seizure frequency. Neurosurgery opinion was sought and she has been advised video EEG and repeat MRI after one month. No active intervention was advised. Her seizures were completely controlled after 2 days and patient discharged from hospital.

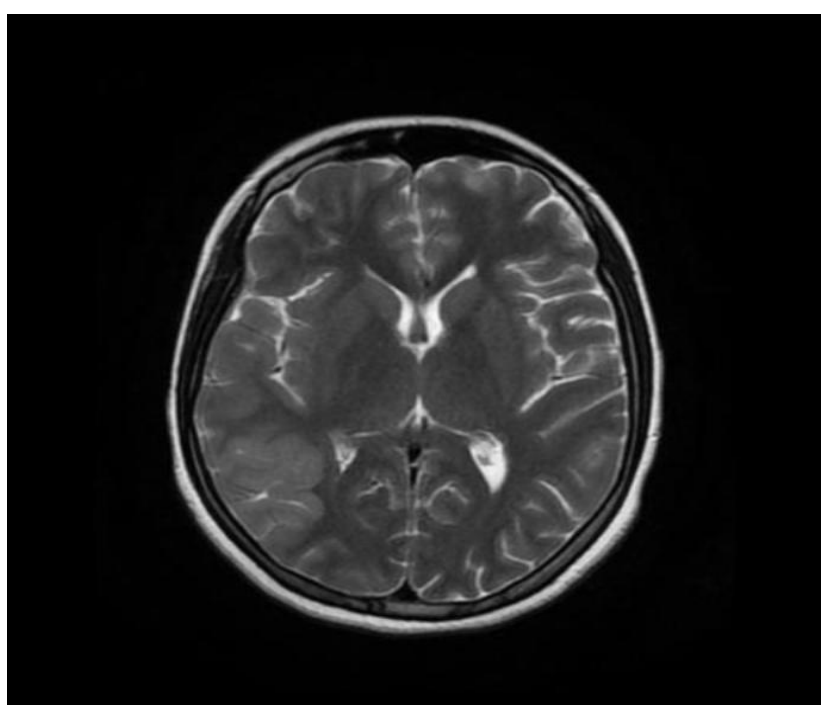

Fig :- 1

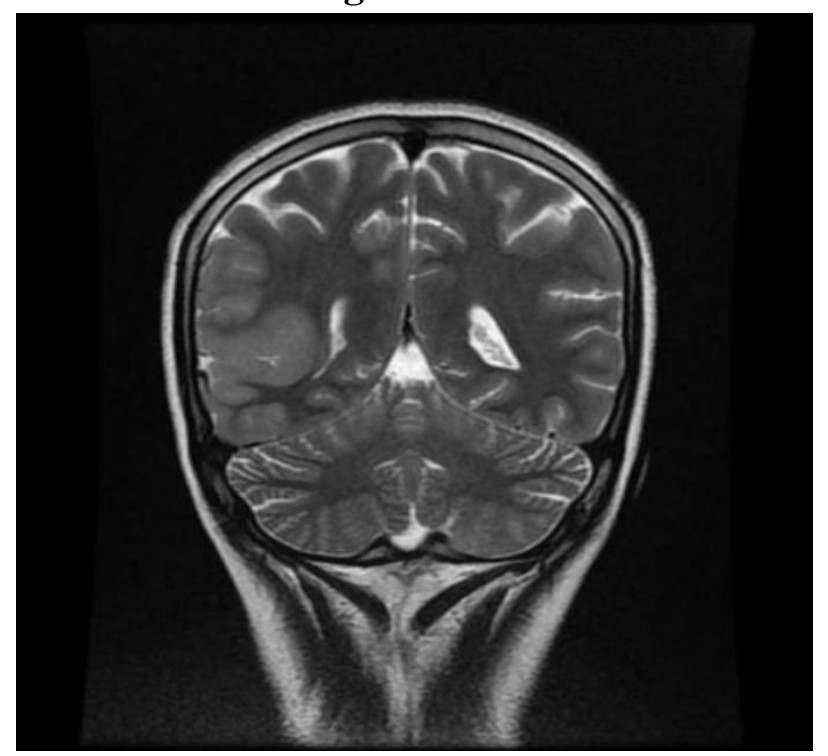

FIG:- 2

Fig 1 \& 2:- MRI brain showed thickened right posterior parietal lobe cortex with iso-intense signal on T1 and T2 weighed images and diffusion restriction and surrounding area of iso intensity on DWI suggesting focal cortical dysplasia with post ictal edema 


\section{Discussion}

Focal cortical dysplasia alludes to the malformations of cortical development that result from pertubations of neuron multiplication, proliferations as well as cortical association ${ }^{(3)}$. Numerous classification have been proposed. In general three types of focal cortical dysplasia are recognised.

Type I: Architecural abnormalities which presents with mild symptomatic expression and late onset is more often seen in adults, with changes present in the temporal lobe.

Type II (Taylor type): Architectural abnormalities and dysplastic neurons.

Type IIa: without balloon cells

Type IIb: with balloon cells.

Malformations of cortical development reflect embryological disruptions in: Neuronal proliferation (ex. TSC), Neuronal migration (ex.heterotopias), Cortical organization (ex.schizencephaly), Multiple steps (ex. focal cortical dysplasia). Clinical symptoms are more severe in type II of cortical dysplasia usually seen in children. In this type more extensive changes occur outside the temporal lobe with predilection for the frontal lobes.

Type III: This type is associated with principal lesion as hippocampal sclerosis, tumor, vascular malformation or acquired pathology during early life. These lesions are important causes of refractory epilepsy $^{(4)}$.

In our case report EEG was normal; it shows EEG is not preferred tool for investigation. MRI findings of our study were similar with other studies ${ }^{(5)}$. MRI is the investigation of choice for malformations of cortical development. More information available from newer sequences and different MR imaging modalities. Neuroimaging of these lesions is important for diagnosis and management decisions in epilepsy, including the feasibility and likely success of surgical resection in cases that are refractory to medication. In this case seizure was controlled by two anticonvulsants.

\section{References}

1. Fauser S, Huppertz HJ, Bast T, Strobl K, Pantazis G, Altenmueller DM, Feil B. Clinical characteristics in focal cortical dysplasia: a retrospective evaluation in a series of120 patients. Brain 2006;129: pp 1907-1916.

2. Saint Martin C, Adamsbaum C, Robain O, Chiron C, Kalifa G. An Unusual Presentation of Focal Cortical Dysplasia. AJNR 1995;16: pp 840-842.

3. Chiang KL, Wong TT, Kwan SY, Hsu TR, Wang $\mathrm{CH}$, Chang HP.Finding on brain MRI mimicking focal cortical dysplasiain early Rasmussen's encephalitis: a case report and review. Childs Nerv Syst 2009:23: pp 5-9.

4. Widdess-Walsh P, Diehl B, NajmI. Neuroimaging of focal cortical dysplaisa. J Neuroimaging 2006;16: pp 185 - 196.

5. Luat AF, Makki M, Chugani HT. Neuroimaging in tuberous sclerosis complex. Curr Op in Neurology 2007;20: pp 142- 150. 